\title{
The $\theta$ parameter in loop quantum gravity: effects on quantum geometry and black hole entropy
}

\author{
Danilo Jimenez Rezende and Alejandro Perez \\ Centre de Physique Théorique*, Campus de Luminy, 13288 Marseille, France.
}

(Dated: October 30, 2018)

\begin{abstract}
The precise analog of the $\theta$-quantization ambiguity of Yang-Mills theory exists for the real $S U(2)$ connection formulation of general relativity. As in the former case $\theta$ labels representations of large gauge transformations, which are super-selection sectors in loop quantum gravity. We show that unless $\theta=0$, the (kinematical) geometric operators such as area and volume are not well defined on spin network states. More precisely the intersection of their domain with the dense set $C y l$ in the kinematical Hilbert space $\mathscr{H}$ of loop quantum gravity is empty. The absence of a well defined notion of area operator acting on spin network states seems at first in conflict with the expected finite black hole entropy. However, we show that the black hole (isolated) horizon area - which in contrast to kinematical area is a (Dirac) physical observable - is indeed well defined, and quantized so that the black hole entropy is proportional to the area. The effect of $\theta$ is negligible in the semiclassical limit where proportionality to area holds.
\end{abstract}

\section{INTRODUCTION}

A remarkable feature of general relativity $(\mathrm{GR})$ is that it admits a connection formulation with a (unconstrained) phase space isomorphic to that of $S U(2)$ Yang Mills theory [1]. This property of GR is of great importance for the definition of the quantization program of loop quantum gravity (LQG). At the basic level, LQG is defined using non perturbative techniques first developed in the context of Yang-Mills theories. For instance, the use of the parallel transport of the $S U(2)$ connection as configuration variable - which combined with diffeomorphism invariance - allows for the precise definition of a (background independent) approach to quantum gravity. The compactness of the associated gauge group lies at the heart of the very existence of the representation of the fundamental operators in a Hilbert space where the constraints of GR can be promoted to finite operators (see [2] and refs. therein).

The $S U(2)$ connection formulation of GR is defined in terms of the so-called AshtekarBarbero variables which are labelled by a real parameter $\gamma$ known as the Immirzi parameter. Here we shall see that a more general $S U(2)$ connection formulation of GR includes a new dimensionless parameter $\theta \in[0,2 \pi]$. These two-parameter family of descriptions of GR are all classically equivalent. However, upon quan-

*Unité Mixte de Recherche (UMR 6207) du CNRS et des Universités Aix-Marseille I, Aix-Marseille II, et du Sud Toulon-Var; laboratoire afilié à la FRUMAM (FR 2291) tization the pair $(\gamma, \theta)$ labels unitarily inequivalent theories and therefore represent a quantization ambiguity of LQG.

In the following section we show how the two parameter family of $S U(2)$ connection formulations can be obtained from a series of canonical transformations starting from the standard ADM or metric Hamiltonian formulation of GR. This implies that all these formulations are classically equivalent.

In Section [II] we show how the newly introduced $\theta$ parameter labels unitary representations of large $S U(2)$ gauge transformations which are super-selected sectors of quantum gravity.

In Section IV we study the implications of the $\theta$ ambiguity for the definition of (kinematical) quantum geometric operators such as area and volume. We show that the action of the latter is not well defined on spin network states. We conjecture that their domain is not dense in the Hilbert space $\mathscr{H}$ of LQG. This might seem problematic if one would like to attribute a physical meaning to kinematical quantities. Since, in LQG the quantization of the area operator plays an important role in the computation of black hole entropy [9, 10], one might expect at first sight difficulties with the computation of black hole entropy for $\theta \neq 0$.

However, in contrast with kinematical area, the black hole horizon area (which is a true observable in the classical theory) can be quantized and has a discrete spectrum in the $\theta \neq 0$. In Section $\mathrm{V}$ we show how the standard result for the black hole entropy is recovered in the semiclassical limit. In Section VI we interpret this result. 


\section{MORE GENERAL CONNECTION FORMULATION OF GR}

The $S U(2)$ Ashtekar-Barbero variables can be introduced in several ways. Perhaps the shortest path is the one defined by a series of canonical transformations starting from the Arnowitt, Deser, and Misner (ADM) variables for general relativity. The ADM parametrization of the (unconstrained) phase space of gravity is given by the canonical pair $\left(q_{a b}, P^{a b}\right)$ with $a, b=1,2,3$ space indices. The configuration variable $q_{a b}$ is the 3 -metric of a Cauchy surface $\Sigma$ (the space time manifold $\mathscr{M}$ is assumed to be of topology $\mathscr{M}=\Sigma \times \mathbb{R}$ ), while the momentum $P^{a b}$ is defined in terms of the extrinsic curvature $K_{a b}$ (Lie derivative of $q_{a b}$ in the direction normal to $\Sigma$ ) as

$$
P^{a b}=q^{-1 / 2}\left(K^{a b}-K q^{a b}\right),
$$

where $q^{a b}$ is the inverse of $q_{a b}, q=\operatorname{det}\left(q_{a b}\right)$, and $K=q_{a b} K^{a b}$.

One can introduce a (densitized) triad defined by the equation

$$
q q^{a b}=E_{j}^{a} E_{i}^{b} \delta^{i j} .
$$

With $E_{i}^{a}$ at hand one introduces the quantity

$$
K_{a}^{i}=q^{-1 / 2} K_{a b} E_{j}^{b} \delta^{i j} .
$$

It is easy to show that-provided $E_{i}^{a}$ and $K_{b}^{j}$ satisfy the constraint [15]

$$
G_{i}=\epsilon_{i j k} E^{a j} K_{a}^{k} \approx 0
$$

- one can express the ADM variables in terms of the pair $E_{i}^{a}$ and $K_{a}^{i}$, and that the latter are indeed canonically conjugate variables.

The Ashtekar-Barbero variables are obtained by the canonical transformation

$$
{ }^{\gamma} P_{i}^{a}=\gamma^{-1} E_{i}^{a} \quad{ }^{\gamma} A_{a}^{i}=\Gamma_{a}^{i}(E)+\gamma K_{a}^{i},
$$

where $\Gamma_{a}^{i}$ is the torsion free spin connection (i.e. a functional of $E_{i}^{a}$ alone), and $\gamma \in \mathbb{R}$ is an arbitrary parameter known as the Immirzi parameter. It is easy to see that the previous transformation is a canonical transformation [3] if one realizes that $\Gamma_{a}^{i}=\delta W_{1}[E] / \delta E_{i}^{a}$ with

$$
W_{1}[E]=\int_{\Sigma} \epsilon_{b c d} E_{[i}^{a} E_{j]}^{b} \partial_{a} \frac{E^{c i} E^{d j}}{\operatorname{det}(E)} .
$$

In terms of the new variables the constraint (3) becomes the Gauss law of $S U(2)$ Yang-Mills theory, namely

$$
G_{i}=D_{a}{ }^{\gamma} P_{i}^{a} \approx 0,
$$

where $D_{a}$ is the covariant derivative defined in terms of the connection ${ }^{\gamma} A_{a}^{i}$.

Are there more general connection variables than the ones obtained above? Yes, if we are given a background independent functional $W_{2}\left[{ }^{\gamma} A\right]$, since one can leave ${ }^{\gamma} A$ unchanged and define a new momentum variable $\left({ }^{\gamma \theta} P_{i}^{a}\right)=$ ${ }^{\gamma} P_{i}^{a}+\delta W_{2}\left[{ }^{\gamma} A\right] / \delta^{\gamma} A_{a}^{i}$. In three dimensions there is only a one parameter family of $\left.W_{2}{ }^{\gamma} A\right]$ given by

$$
W_{2}=\frac{\theta}{16 \pi^{2}} \int_{\Sigma} \operatorname{Tr}\left[{ }^{\gamma} A \wedge d^{\gamma} A+\frac{2}{3}{ }^{\gamma} A \wedge^{\gamma} A \wedge^{\gamma} A\right],
$$

where $\theta$ is a real parameter and the integral is the well known Chern-Simons action. In terms of the canonical pair $\left(E_{a}^{i}, K_{b}^{j}\right)$ we get

$$
{ }^{\gamma \theta} P_{i}^{a}=\gamma^{-1} E_{i}^{a}+\frac{\theta}{8 \pi^{2}} B_{i}^{a} \quad{ }^{\gamma} A_{a}^{i}=\Gamma_{a}^{i}+\gamma K_{a}^{i},(8)
$$

where $B_{i}^{a}=\epsilon^{a b c} F_{b c}^{j} \delta_{i j}$ is the non Abelian magnetic field of $\gamma A_{a}^{i}$. Due to the Bianchi identity the Gauss constraint has the same functional form as (6) where ${ }^{\gamma} P$ is replaced by ${ }^{\gamma \theta} P$. From the remaining constraints only the Hamiltonian or scalar constraint has an explicit $\theta$-dependent $\mathrm{P}$ violating term [4] (see also [5]).

\section{LARGE $S U(2)$ GAUGE TRANSFORMATIONS}

There is a nice geometric way of understanding the previous quantization ambiguity [4] (see also [6] and [7] for a similar argument and analogies concerning the origin of $\gamma$ ). In the quantum theory states are required to be anihilated by the quantum Gauss constraint. As the latter is the infinitesimal generator of local $S U(2)$ transformations, states are required to be invariant under the gauge group $\mathscr{G}_{0}$ of transformations connected to the identity. However, assuming for the moment that $\Sigma$ is compact, and due to the fact that $\pi_{3}(S U(2))=\mathbb{Z}$, the full gauge group of gravity $\mathscr{G}$ also contains the socalled large gauge transformations. The latter are gauge transformation $g(x)$ with non trivial winding number

$$
w[g]=\frac{1}{24 \pi^{2}} \int \operatorname{tr}\left[g^{-1} d g \wedge g^{-1} d g \wedge g^{-1} d g\right] .
$$

Now if $\alpha \in \mathscr{G}$ is such that $w[\alpha]=1$ then one can show that $\mathscr{G} / \mathscr{G}_{0} \approx\left\{\alpha^{n} / n \in \mathbb{Z}\right\}$. Therefore, physical states can transform in a non trivial fashion under large gauge transformations. We denote $\mathscr{H}_{\theta}$ the unitary (irreducible) representations of $\mathscr{G} / \mathscr{G}_{0} \approx \mathbb{Z}$ which are labelled by an 
angle $\theta \in[0,2 \pi]$. Given $\Psi \in \mathscr{H}_{\theta}$ and $\alpha \in \mathscr{G}$ (s.t. $w[\alpha]=1$ )

$$
\alpha \triangleright \Psi=e^{i \theta} \Psi .
$$

Finally due to the fact that physical observables are invariant under the full group $\mathscr{G}$ they leave $\mathscr{H}_{\theta}$ invariant. Hence, each $\mathscr{H}_{\theta}$ gives a different quantization.

The relationship with the analysis of the previous section is clarified if one realizes that the non trivial transformation rule for states in $\mathscr{H}_{\theta}$ can be shifted to operators by means of the state redefinition

$$
\tilde{\Psi}[A]=\exp \left(-i W_{2}[A]\right) \Psi[A]
$$

with $W_{2}[A]$ as defined in (77) [16]. Hence working with wave functions with non trivial behaviour under large gauge transformation is equivalent to working with a transformed momentum

$$
{ }^{\gamma \theta} P_{i}^{a}=\exp \left(-i W_{2}[A]\right)^{\gamma} P_{i}^{a} \exp \left(i W_{2}[A]\right),
$$

which has the form (8) on $\mathscr{H}_{0}=\mathscr{H}$ (spanned by spin network states). Therefore, the $\theta$ ambiguity, described in Section [, has a clear meaning in the quantum theory. It encodes the non trivial representations of $\mathscr{G} / \mathscr{G}_{0}$ which are super selected sectors of quantum gravity.

\section{QUANTUM GEOMETRY}

In the standard LQG formulation of the quantum theory [2] the fundamental (to-bequantized) variables are the holonomy of ${ }^{\gamma} A$ along one dimensional paths $e \subset \Sigma$, and fluxes of ${ }^{\gamma \theta} P$ across surfaces $S \subset \Sigma$. Respectively,

$h_{e}\left[{ }^{\gamma} A\right]=\mathrm{P} e^{-\int_{e}^{\gamma^{\gamma} A}}$ and ${ }^{{ }^{\theta}} P(r, S)=\int_{S} r \cdot\left(\epsilon^{\gamma \theta} P\right)$,

where the 2-form $r \cdot \epsilon^{\gamma} P_{a b}=r^{i} \epsilon_{a b c}{ }^{\gamma} P_{i}^{c}$, and $r^{i}$ is an arbitrary field of internal directions. These (kinematical) observables can be represented as self adjoint operators in the (kinematical) Hilbert space $\mathscr{H}$ of LQG. The fundamental excitations in $\mathscr{H}$ are given by quantized lines of flux of ${ }^{\gamma \theta} P_{i}^{a}$ which can be organized in an orthonormal basis of (open) spin network states. The quantum flux operators ${ }^{\gamma \theta} P(r, S)$ have discrete spectrum. More precisely, the basic non trivial quanta of ${ }^{\gamma \theta} P(r, S)$ are given by a spin network edge labelled by the spin $j$ and magnetic number $m$ transversal to $S$ and ending at $S$. In that case the possible values of this puncture-like contribution to ${ }^{\gamma \theta} \mathrm{P}(r, S)$ is $8 \pi \ell_{p}^{2} m$ for $-j \leq m \leq j$. The general eigenstates are given by states with $n$ punctures $\left|n,\left\{j_{i}, m_{i}\right\}_{i=1}^{n}\right\rangle$ for which

$$
\left({ }^{\gamma \theta} P(r, S)-8 \pi \ell_{p}^{2} \sum_{i=1}^{n} m_{i}\right)\left|n ;\left\{j_{i}, m_{i}\right\}_{i=1}^{n}\right\rangle=0,(11
$$

with $-j_{i} \leq m_{i} \leq j_{i}$. The position of the punctures on $S$ also labels the previous states. We have simplified the notation here as the details are not essential for our argumentation.

There are two simple $S U(2)$ invariant operator that one can construct (both having discrete spectra [2]). The first one associates to any surface $S \subset \Sigma$ the quantity

$$
O_{1}\left(S,{ }^{\gamma \theta} P\right)=\int_{S} \sqrt{\left({ }^{\gamma \theta} P_{i}^{a}\right)\left({ }^{\theta} P_{j}^{b}\right) \delta^{i j} n_{a} n_{b}},
$$

where $n_{a}$ is the normal to the surface $S$. The other one associates to a three dimensional region $U \subset \Sigma$ the quantity

$$
O_{2}\left(U,{ }^{\gamma \theta} P\right)=\int_{U} \sqrt{\left({ }^{\gamma \theta} P_{i}^{a}\right)\left({ }^{\gamma \theta} P_{j}^{b}\right)\left({ }^{\theta} P_{k}^{c}\right) \epsilon^{i j k} \epsilon_{a b c}}
$$

However, according to (1) the field encoding the dynamical Riemannian geometry of $\Sigma$ is

$$
E_{i}^{a}=\gamma\left({ }^{\gamma \theta} P_{i}^{a}\right)-\frac{\theta}{8 \pi^{2}} \gamma B_{i}^{a}\left({ }^{\gamma} A\right) .
$$

Therefore, in the case $\theta=0$ the previous operators have a clear geometric meaning: $O_{1}$ relates to the area of $S$ via $\mathcal{A}(S)=\gamma O_{1}(A)$, and $O_{2}$ relates to the volume of $U$ via $\mathcal{V}(U)=$ $\gamma^{\frac{3}{2}} O_{2}(U, P)$. However, for $\theta \neq 0$, the area and volume depend on both the connection ${ }^{\gamma} A$ (through the magnetic field $B_{i}^{a}$ ) and ${ }^{\gamma \theta} P$. In that case these operators are not well defined in the kinematical hilbert space of LQG (see Appendix for an explicit proof of this statement).

Here, the term kinematical observable is used to designate quantities that are not measurable in the theory but serve to setup Dirac's quantization program. The reason for that is that the (kinematical) observables introduced above are not invariant under the full gauge group of GR that includes diffeomorphisms in addition to local $S U(2)$. This qualification is quite important for the interpretation of our results. We shall come back to this in Section VI once we elaborate a bit more on the effects of $\theta$.

\section{BLACK HOLE ENTROPY}

In Section IV] we argued that the discreteness of kinematic geometric operators is lost in the $\theta \neq 0$ sectors. Despite of this fact we show here 
that (due to dynamical effects) the quantum operator associated to the area of black hole (isolated) horizon has indeed a discrete spectrum for arbitrary $\theta$. This leads to a finite entropy of the black hole horizon which is proportional to the macroscopic area. $\theta$ does not affect the leading order contribution to the entropy calculation.

The computation of Black Hole entropy is based on the quantization of a sector of the phase space of GR containing a so-called isolated horizon (IH) 9] (for simplicity here we will assume the horizon is non rotating). Physically a non rotating $\mathrm{IH}$ is a three dimensional null surface $\Delta$ equipped with a preferred foliation by 2 -spheres such that the area of the spheres is constant.

The boundary conditions on the horizon reduce the $S U(2)$ gauge symmetries on the bulk to a $U(1)$ subgroup leaving invariant an internal direction $r^{i}$. There are non trivial degrees of freedom at the horizon encoded in the pull back of the bulk connection on the horizon $H=\Delta \cap \Sigma$; a $U(1)$-connection ${ }^{\gamma} A={ }^{\gamma} A^{i} r_{i}$ (notice the obvious abuse of notation). The validity of Einstein's equations at the horizon imply the following relationship between bulk and horizon degrees of freedom:

$$
F_{a b}\left({ }^{\gamma} A\right)=-\frac{2 \pi}{a_{H}} \epsilon_{a b c} E^{c}{ }_{i} r^{i},
$$

where $a_{H}$ is the macroscopic area of the horizon.

As shown in [9] the IH boundary condition consistently defines a sector of the phase space of gravity. The requirement that the simplectic structure be foliation independent introduces a Chern-Simons boundary term. The latter leads to the quantization of the degrees of freedom on the horizon. More precisely, the simplectic potential in terms of the variables $\left({ }^{\gamma} P,{ }^{\gamma} A\right)$ is

$$
\begin{aligned}
& 8 \pi G^{\gamma} \Theta(\delta)= \\
& -\int_{\Sigma} \operatorname{Tr} \delta^{\gamma} A \wedge \epsilon^{\gamma} P+\frac{a_{H}}{4 \pi \gamma} \int_{H} \delta^{\gamma} A \wedge^{\gamma} A .
\end{aligned}
$$

In order to express it in terms of (8) one adds to ${ }^{\gamma} \Theta(\delta)$ the 'total field differential' of $W_{2}[A]$;

$$
\delta W_{2}\left[{ }^{\gamma} A\right]=\theta\left(2 \int_{\Sigma} \operatorname{Tr} F\left({ }^{\gamma} A\right) \wedge \delta^{\gamma} A+\int_{H}{ }^{\gamma} A \wedge \delta^{\gamma} A\right),
$$

and the new potential becomes

$$
\begin{aligned}
{ }^{\gamma} \Theta & = \\
& =-\frac{\hbar}{\ell_{p}^{2}} \int_{\Sigma} \operatorname{Tr} \delta^{\gamma} A \wedge \epsilon^{\gamma \theta} P+\kappa \hbar \int_{H} \delta^{\gamma} A \wedge{ }^{\gamma} A,
\end{aligned}
$$

where $\kappa=a_{H} /\left(4 \pi \gamma \ell_{p}^{2}\right)-\theta / 16 \pi^{2}$. So we see that in addition to the $\theta$ modification (8) of the momentum conjugate to ${ }^{\gamma} A$ in the bulk, the canonical transformation has the effect of shifting the level $\kappa$ of the Chern-Simons contribution to the simplectic structure. In terms of the phase space variables the (dynamical) constraint (13) takes the simple form

$$
2 \kappa F\left({ }^{\gamma} A\right)=-\epsilon^{\gamma \theta} P^{i} r_{i} .
$$

Remarkably the constraint (16) has the same functional form as the one found in 9] for the $\theta=0$ case. The $\theta$-dependence is hidden in the explicit form of the Chern-Simons level $\kappa$ (A similar thing happens in the quantization of $\mathrm{IH}$ in the presence of non minimally coupled scalar fields [11]). This implies that the quantization techniques of [9] can be directly applied to the $\theta \neq 0$ case. In the quantum theory the above requirement restricts the set of physical states. The constraint (16) requires states in the bulk to be eigen-states of the flux of ${ }^{\gamma \theta} \mathrm{P} \cdot r$ across $\mathrm{H}$. These are spin network edges carrying spins $j$ ending at the horizon such that they are eigenstates of the r.h.s. of (16). From (11), the allowed eigenvalues of a single puncture are $8 \pi l_{p}^{2} m$ with $-j \leq m \leq j$. On the other hand, the quantization of Chern-Simons implies that the holonomy around a single puncture has eigenvalues $\exp i 2 \pi a_{i} / \kappa$ with $a_{i} \in \mathbb{Z} \bmod \kappa$. Quantum Einstein eqs. (16) select those states for which $a_{i}=-2 m_{i} \bmod \kappa$. Moreover, states satisfying (16) are eigenstates of the quantum horizon area. Explicitly one has

$$
\begin{aligned}
& A_{H}\left|n ;\left\{j_{i}, m_{i}\right\}_{i=1}^{n}\right\rangle \\
& =8 \pi \gamma \ell_{p}^{2} \sum_{i=1}^{n} \sqrt{K m_{i}^{2}+j_{i}\left(j_{i}+1\right)}\left|n,\left\{j_{i}, m_{i}\right\}_{i=1}^{n}\right\rangle,
\end{aligned}
$$

where $K=\theta\left(a_{H} /\left(4 \pi \gamma \ell_{p}^{2}\right)-\theta / 16 \pi^{2}\right)^{-1}, n$ is the number of punctures, and $\left\{j_{i}, m_{i}\right\}_{i=1}^{n}$ is the set of quantum numbers associated to the punctures on $H$. Notice that discreteness follows from the quantization of the magnetic flux at $H$ (compare with the generalized eigenstate defined in the Appendix). Therefore, here the dynamical condition (16) implies that the quantum operator associated to the Dirac observable $A_{H}$ is well defined. Using the counting techniques of [12] one finds that the $\theta$ dependence does not change the leading term in the entropy: explicitly $S_{H}:=\log \left[\mathscr{N}\left(a_{H}\right)\right] \approx$ $\left(4 \ell_{p} \gamma\right)^{-1} \gamma_{M} a_{H}$, where $\mathscr{N}\left(a_{H}\right)$ is the number of horizon states compatible with a macroscopic horizon area $a_{H}$ and $\gamma_{M}=0.23 \ldots$ 


\section{DISCUSSION}

General relativity admits a two-parameter family of $S U(2)$ connection formulations labelled by the Immirzi parameter $\gamma \in \mathbb{R}$ and $\theta \in[0,2 \pi]$. Our arguments show that discreteness of (kinematical) geometric operators in LQG is a special property of the $\theta=0$ sector. For $\theta \neq 0$ kinematical area and volume are far more complicated objects. In the appendix we prove some properties that suggest that they are not densely defined in $\mathscr{H}$ (which we conjecture). Nevertheless, discreteness at the fundamental level remains in the sense that the (kinematic) Hilbert space is given by the span of spin network states (labelled graphs and discrete quantum numbers).

What are the implications of this underlying discrete structure? General covariance implies that only fully gauge invariant observables (i.e. Dirac observables which are both $S U(2)$ and diffeomorphism invariant) are physically meaningful. From this perspective the discreteness of kinematical area and volume (although an interesting property when present) is not by itself of direct physical relevance. The physical question is rather whether the fundamental discreteness of the state space of LQG would leave imprints in fully gauge invariant quantities which represent physical observables. Unfortunately, due to the dynamical nature of these observables, this question is very difficult to answer in general at this stage of development of LQG.

Nevertheless, one can try to answer the question in particular cases. One such case is the $\mathrm{IH}$ system, where the black hole area is a Dirac observable. From our viewpoint this is the most important result of the paper: we have shown that due to the dynamics of general relativityencoded in the IH boundary condition - the spectrum of the area of black hole horizon remains discrete. In this case problems concerning the quantization of (kinematical) area can be viewed as a gauge artifact that disappear when looking at gauge invariant IH area.

As in $\mathrm{QCD}$, the angle $\theta$ introduces parity (as well as time reversal) violation in quantum gravity [4] 17]. As a consequence, one would expect only $P$ violating observables to be sensitive to $\theta$. The black hole IH system is such an example: notice for instance the $P$-violating nature of the IH boundary condition (16). Therefore the $\theta$-dependent effects found here are expected from general considerations. As in the quantum theory $\theta$ is defined modulo $2 \pi$ the latter effects are sub-leading terms that are not relevant in the semiclassical limit $\left(a_{H} / \ell_{p} \gg 1\right)$. Our results represent another non trivial test for the uni- versality $\mathrm{BH}$ entropy in loop quantum gravity.

As explained in Section [II the Gauss and vector constraints of canonical gravity are unchanged in the $\theta \neq 0$ sectors. However, the scalar constraint now has new P-violating terms. We have concentrated here on a very specific dynamical situation where the precise form of the scalar constraint did not play any important role (in essence the scalar constraint is replaced by the condition (16) when dealing with IHs). It would be interesting to investigate the effects of the additional terms to the dynamics of LQG (of special interest is the case where fermions are present).

The quantization of the (kinematical) volume operator plays a central role in the quantization of the scalar constraint in the $\theta=0$ sector 14]. The difficulties associated with the quantization of the (kinematical) area operator described in the appendix would also appear in the quantization of volume in the $\theta \neq 0$ sectors. In this sense, it seems that the usual quantization techniques applied to the scalar constraint cannot be imported directly to the generic sectors. This issue should be studied in detail.

\section{Acknowledgements}

This work was supported in part by: the Agence Nationale de la Recherche, Grant No. ANR-06-BLAN-0050. Thanks to A. Corichi, J. Engle, M. Knecht, J. Lewandowski, M. Montesinos, J. Pullin, C. Rovelli, H. Sahlmann and T. Thiemann for discussions.

\section{APPENDIX A: ON THE QUANTIZATION OF GEOMETRIC OPERATORS}

Here we argue that the kinematical geometric operators are not well defined in the $\theta \neq 0$ case. We do so by showing explicitly that the action of the area operator is ill defined on elements of $C y l \subset \mathscr{H}$.

Without loss of generality we can assume that we have local coordinates $x^{1}, x^{2}, x^{3}$ and that the surface $S$ is defined by $x^{3}=0$. In terms of our basic phase space variables the area $A(S)$ takes the form

$$
\begin{aligned}
& A(S)=\int \sqrt{E^{3} \cdot E^{3}} \\
& =\gamma \int \sqrt{\left(P^{3}-\frac{\theta}{8 \pi^{2}} B^{3}\right) \cdot\left(P^{3}-\frac{\theta}{8 \pi^{2}} B^{3}\right)} .
\end{aligned}
$$

In order to quantize the previous expression one needs to introduce a regularization. We 
take for example a cellular decomposition of $S$. As it will become clear below the details of the regularization do not matter for our argument. Therefore, here we take the dual of the lattice $(\epsilon n, \epsilon m, 0)$ for $0<\epsilon \in \mathbb{R}$ and $n, m \in \mathbb{Z}$. Then at the classical level it is easy to see that

$$
A(S)=\lim _{\epsilon \rightarrow 0} \sum_{n, m} \sqrt{E\left(S^{n m}, \tau^{i}\right) E\left(S^{n m}, \tau_{i}\right)}
$$

where $S^{n m}$ is the plaquette dual to the lattice point $\epsilon(n, m, 0)$, and

$$
E\left(S^{n m}, \tau^{i}\right)=\gamma P\left(S^{n m}, \tau^{i}\right)-2 \gamma \theta B\left(S^{n m}, \tau^{i}\right)
$$

with

$$
B\left(S, r^{i}\right):=\int B_{i}^{3} r^{i} d x^{1} d x^{2}
$$

and $P\left(S^{n m}, \tau^{i}\right)$ defined in Section [V] Let us concentrate for the moment on the action of the regularized area operator on the constant function $1 \in C y l$. Using the fact that $P\left(S^{n m}, \tau_{i}\right) \triangleright 1=0$ for all $n, m$ we have:

$$
\begin{aligned}
& \lim _{\epsilon \rightarrow 0} \sum_{n, m} \sqrt{E\left(S^{n m}, \tau^{i}\right) E\left(S^{n m}, \tau_{i}\right)} \triangleright 1= \\
& =\lim _{\epsilon \rightarrow 0} \sum_{n, m} \sqrt{B\left(S^{n m}, \tau^{i}\right) B\left(S^{n m}, \tau_{i}\right)} \triangleright 1 \\
& =2 \lim _{\epsilon \rightarrow 0} \sum_{n, m} \sqrt{\operatorname{Tr}\left[U^{n m} \tau_{i}\right] \operatorname{Tr}\left[U^{n m} \tau^{i}\right]} \triangleright 1,
\end{aligned}
$$

where we used that

$$
B\left(S^{n m}, \tau_{i}\right)=2 \operatorname{Tr}\left[U^{n m} \tau_{i}\right]+\mathcal{O}\left(\epsilon^{4}\right)
$$

in the last line. Notice that the last line implies that the action on $1 \in C y l$ is given by a sum of mutually-orthogonal terms in $C y l \subset \mathscr{H}$. Therefor the norm of such regularized action grows with the number of plaquettes and we have

$$
\left\|\sum_{n, m} \sqrt{\operatorname{Tr}\left[U^{n m} \tau_{i}\right] \operatorname{Tr}\left[U^{n m} \tau^{i}\right]} \triangleright 1\right\| \sim \frac{1}{\epsilon^{2}} .
$$

We conclude that the action of $A(S) \triangleright 1$ is not defined. From this one can easily see that the same problem with the limit $\epsilon \rightarrow 0$ arises for any $\phi \in C y l$ : for an arbitrary $\phi \in C y l$ it suffices to concentrate on an open region of $S$ that does not intersect the graph defining $\phi$. This problem is not surprising, it is completely equivalent to the one found if one tries to define for example the Yang-Mills hamiltonian in $\mathscr{H}$. The action of the area operator on $C y l$ is not well defined in the $\theta \neq 0$ sectors.
Is the domain of the area operator dense in $\mathscr{H}$ ? It seems that the only way to avoid the divergences found above one would need the plaquette actions in the regulated operator to act trivially. Formally speaking one would need the 'quantum' magnetic field to vanish almost everywhere on $S$. Such states are however outside of $\mathscr{H}$ and can only be given a distributional meaning. From this it seems that it is reasonable to conjecture that the area operator is not even densely defined in $\mathscr{H}$. A similar conclusion can be obtained for the volume.

For completeness we give here an example of distributional states $\Psi$ in the dual space $C y l^{\star}$ for which the action of $A(S)$ is well defined in the sense that $A(s) \triangleright \Psi \in C y l^{\star}$ where

$$
A(s) \triangleright \Psi(\phi):=\Psi(A(s) \triangleright \phi) \quad \forall \phi \in C y l .
$$

We shall do this by exhibiting a special family of states $\Psi_{a} \in C y l^{\star}$ labelled by a group element $a \in S U(2)$. The state is defined by its action on $C y l$. It would suffice to define the action of $\Psi_{a}$ on any element of the spin network basis.

Definition: For any $\phi \in C y l$ we define the action of $\Psi_{a}$ by the following three properties:

1. $\Psi_{a}(1)=1$

2. $\Psi_{a}(\phi)=0$ if the underlying graph of $\phi$ is not entirely contained on $S$ (in our coordinates if it does not lie on the $x^{3}=0$ plane)

3. If the graph of $\phi$ is contained in $S$ then we decompose $\phi$ in terms of spin networks, and subsequently we write the spin networks as a product of Wilson loops. In this way we can write any such element of $\phi \in C y l$ as

$$
\phi=\sum_{L} c_{L} \prod_{\ell \in L} \alpha_{\ell}
$$

where $L$ are collections of loops, $\ell \in L$ denotes a loop in the collection $L$ and $\alpha_{\ell}$ is the trace of the holonomy in the fundamental representation around $\ell$. The loops $\ell$ are all contained in $S$, and can have self-intersections. We define

$$
\Psi_{a}(\phi):=\sum_{L} c_{L} \prod_{\ell \in L} \Psi_{a}\left(\alpha_{\ell}\right),
$$

where $\Psi_{a}\left(\alpha_{\ell}\right)=\operatorname{tr}\left(a^{w[\ell]}\right)$ where $w[\ell]$ is the winding number of $\ell$ around the point $(0,0,0)$.

Lemma: For $S^{I} \subset S$, any The following identities follow from the previous definition

$$
\Psi_{a}\left(P\left(S^{I}, \tau^{i}\right) P\left(S^{I}, \tau_{i}\right) \triangleright \phi\right)=0,
$$


and

$$
\begin{aligned}
& \Psi_{a}\left(P\left(S^{I}, \tau^{i}\right) B\left(S^{I}, \tau_{i}\right) \triangleright \phi\right)= \\
& \quad=\Psi_{a}\left(B\left(S^{I}, \tau_{i}\right) P\left(S^{I}, \tau^{i}\right) \triangleright \phi\right)=0,
\end{aligned}
$$

or all $\phi \in C y l$.

Proof: If $\phi$ can be expanded in terms of spin networks fully contained in $S$ then the implication of the lemma is obvious because $P^{3}$ commutes with $B^{3}$ and annihilates such states. In order to avoid the trivial action of $P^{3}$ we need spin network edges that are transversal to the surface $S$. In that case the action of $P^{3}$ is non trivial but the resulting state in both A7) and (A8) contains edges that are outside $S$ and therefore the implication of the lemma follows from the condition (2) of our definition.

Proposition: The (generalized) state $\Psi_{a}$ is a (generalized) eigenstate of the area $A(S)$ with eigenvalue $\gamma \frac{\theta}{4 \pi^{2}} \sqrt{a_{i} a^{i}}$, where $a_{i}=\operatorname{tr}\left(a \tau_{i}\right)$.

Proof: We start from the regularized expresion (A8) and concentrate for a moment on the argument of the square root for the term $(n, m)$.
Using the lemma above we have

$$
\begin{aligned}
& \Psi_{a}\left(E\left(S^{n m}, \tau_{i}\right) E\left(S^{n m}, \tau^{i}\right) \triangleright \phi\right)= \\
& \quad=\left(\frac{\theta}{8 \pi^{2}}\right)^{2} \Psi_{a}\left(B\left(S^{n m}, \tau_{i}\right) B\left(S^{n m}, \tau^{i}\right) \triangleright \phi\right)
\end{aligned}
$$

finally using (A3) we get

$$
\begin{aligned}
& \Psi_{a}\left(E\left(S^{n m}, \tau_{i}\right) E\left(S^{n m}, \tau^{i}\right) \triangleright \phi\right)= \\
& =\left(\frac{\theta}{4 \pi^{2}}\right)^{2} \Psi_{a}\left(\operatorname{tr}\left[U^{n m} \tau_{i}\right] \operatorname{tr}\left[U^{n m} \tau^{i}\right] \triangleright \phi+\mathcal{O}\left(\epsilon^{4}\right)\right)= \\
& \quad=\left(\frac{\theta}{4 \pi^{2}}\right)^{2} \delta_{n 0} \delta_{m 0} a_{i} a^{i} \Psi_{a}(\phi)+\mathcal{O}\left(\epsilon^{4}\right), \quad \text { (A9) }
\end{aligned}
$$

where $a_{i}=\operatorname{Tr}\left(a \tau_{i}\right)$. In the previous equation we used the fact that for $(n, m) \neq(0,0)$ $\left(\operatorname{tr}\left[U^{n m} \tau_{i}\right] \operatorname{tr}\left[U^{n m} \tau_{i}\right]\right)=\left(\operatorname{Tr}\left[\tau_{i}\right] \operatorname{Tr}\left[\tau_{i}\right]\right)=0$. Putting all this together we ntice that we can in this case take the limit $\epsilon \rightarrow 0$. The result is

$$
\Psi_{a}(A(S) \triangleright \phi)=\frac{\theta}{4 \pi^{2}} \gamma \sqrt{a \cdot a} \Psi_{a}(\phi),
$$

for all $\phi \in C y l$. Our generalized state $\Psi_{a}$ is an eigenstate of the area with an eigenvalue that varies continuously as $a \in S U(2)$ varies.
[1] A. Ashtekar, Phys. Rev. D 36 (1987) 1587. J. F. Barbero, Phys. Rev. D 49 (1994) 6935 arXiv:gr-qc/9311019.

[2] T. Thiemann, "Modern Canonical Quantum GR," Cambridge, UK: Univ. Pr. (2007). C. Rovelli, "Quantum gravity," Cambridge, UK: Univ. Pr. (2004) 455 p. C. Rovelli, " Quantum gravity," Cambridge, UK: Univ. Pr. (2004) 455 p. A. Ashtekar and J. Lewandowski, "Background independent quantum gravity: A status report," Class. Quant. Grav. 21, R53 (2004) arXiv:gr-qc/0404018. A. Perez, "Introduction to loop quantum gravity and spin foams," Proceedings of the International Conference on Fundamental Interactions, Domingos Martins, Brazil, (2004) arXiv:gr-qc/0409061.

[3] T. Thiemann, Class. Quant. Grav. 13 (1996) 1383 arXiv:gr-qc/9511057.

[4] A. Ashtekar, A. P. Balachandran and S. Jo, Int. J. Mod. Phys. A 4 (1989) 1493.

[5] M. Montesinos, Class. Quant. Grav. 18 (2001) 1847 arXiv:gr-qc/0104068.

[6] S. Mercuri, arXiv:0708.0037] [gr-qc].

[7] R. Gambini, O. Obregon and J. Pullin, Phys. Rev. D $59 \quad$ (1999) 047505 arXiv:gr-qc/9801055.

[8] H. Kodama, Phys. Rev. D 42 (1990) 2548. L. Smolin, arXiv:hep-th/0209079. L. Freidel and L. Smolin, Class. Quant. Grav. 21 (2004) 3831 arXiv:hep-th/0310224.
[9] A. Ashtekar, S. Fairhurst and B. Krishnan, Phys. Rev. D 62, 104025 (2000). A. Ashtekar, J. Baez, A. Corichi and K. Krasnov, Phys. Rev. Lett. 80, 904 (1998). A. Ashtekar, A. Corichi and K. Krasnov, Adv. Theor. Math. Phys. 3, 419 (2000) arXiv:gr-qc/9905089.

[10] C. Rovelli, Phys. Rev. Lett. 77 (1996) 3288 arXiv:gr-qc/9603063.

[11] A. Ashtekar, A. Corichi and D. Sudarsky, Class. Quant. Grav. 20 (2003) 3413 arXiv:gr-qc/0305044. A. Ashtekar and A. Corichi, Class. Quant. Grav. 20 (2003) 4473 arXiv:gr-qc/0305082.

[12] K. A. Meissner, Class. Quant. Grav. 21, 5245 (2004). M. Domagala and J. Lewandowski, Class. Quant. Grav. 21, 5233 (2004).

[13] B. Dittrich and T. Thiemann, arXiv:0708.1721 [gr-qc]. C. Rovelli, arXiv:0708.2481 [gr-qc].

[14] T. Thiemann, "Quantum spin dynamics (QSD)," Class. Quant. Grav. 15 (1998) 839 arXiv:gr-qc/9606089.

[15] This constraint comes from the fact that $K_{a b}=$ $K_{b a}$ and can be shown to generate local $S U(2)$ rotations of the triad canonical variables.

[16] Transformation (10) corresponds to multiplication by the Kodama state. For the relevance of such state for quantum gravity see [8].

[17] Notice that under a parity transformation the magnetic field $B$ changes sign. 